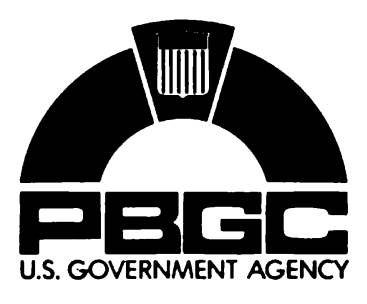

\title{
Pension Benefit Guaranty Corporation
}

\section{Annual Report to the President and Congress \\ lune 30,1975}

